\title{
An unusual complication of pigtail catheter insertion
}

\author{
Amina Saqib', Uroosa Ibrahim², Rabih Maroun ${ }^{1}$ \\ ${ }^{1}$ Department of Pulmonary/Critical Care, ${ }^{2}$ Department of Hematology/Oncology, Staten Island University Hospital, Staten Island, New York, USA \\ Correspondence to: Amina Saqib. Department of Pulmonary/Critical Care, Staten Island University Hospital, Staten Island, New York, USA. \\ Email: saqib.amina@gmail.com.
}

\begin{abstract}
Thoracostomy tubes are indicated for management of air or fluid in the pleural cavity. Pigtail catheters have emerged as an effective and less morbid alternative to traditional large bore chest tubes for evacuation of pleural air or fluid. However, they do not come without complications which commonly include pneumothorax and hemothorax. Rare complications in the literature such as left ventricular penetration, subclavian artery laceration and cerebral air embolism have been reported. We report a case of a 72 -year-old male who presented with dyspnea and was found to have a right-sided pleural effusion requiring thoracentesis and subsequent pigtail catheter placement because of re-accumulation of the fluid. After accidental dislodgement of the catheter, it was replaced and the following day a chest X-ray (CXR) demonstrated diffuse subcutaneous emphysema. Computed tomography (CT) scan of the chest demonstrated the pigtail catheter tracking through the right middle and lower lobes reaching the posterior pleural space. We discuss the implications of this occurrence and recommended management based on our experience.
\end{abstract}

Keywords: Complication; pigtail catheter; lung penetration

Submitted Apr 04, 2018. Accepted for publication May 04, 2018.

doi: $10.21037 /$ jtd.2018.05.65

View this article at: http://dx.doi.org/10.21037/jtd.2018.05.65

\section{Introduction}

Pigtail catheters have emerged as an effective and less morbid alternative to traditional large bore chest tubes for evacuation of pleural air or fluid. The smaller caliber and flexibility of the catheter provides the advantage of less pain and surrounding tissue injury (1). However, the procedure does not come without complications which commonly include pneumothorax and hemothorax. We report an extremely rare complication of lung penetration by the pigtail catheter through two lobes of the right lung.

\section{Case presentation}

A 72-year-old male with a medical history of asthma, gout, hypertension, melanoma excision and umbilical hernia presented to the emergency room with complaints of acute onset progressively worsening shortness of breath. He had a 40-pack-year history of smoking and quit at age 40 . $\mathrm{He}$ had associated symptoms of dry cough and myalgias. On presentation, he was afebrile and saturating $93 \%$ on room air. Physical examination revealed decreased breath sounds over the right base of the lung. Chest X-ray (CXR) demonstrated a right-sided pleural effusion. Computed tomography (CT) of the chest showed a moderate right pleural effusion with overlying compressive atelectasis and superimposed consolidation concerning for a pneumonic process. It also revealed a concentric pericardial effusion (Figure 1).

The patient underwent thoracentesis with drainage of $1,120 \mathrm{~mL}$ of serosanguinous fluid. Fluid analysis was consistent with an exudative pleural effusion. Gram stain, bacterial culture and acid-fast culture were negative. The pleural fluid cytology was positive for malignant cells favoring adenocarcinoma.

Two days later, the patient reported dyspnea on exertion and orthopnea. CXR was consistent with re-accumulation of the pleural fluid requiring a 14 French pigtail catheter insertion under ultrasound guidance. One thousand and seven hundred $\mathrm{mL}$ of cloudy serosanguinous fluid was drained at the time of insertion. Appropriate positioning of the pigtail catheter was confirmed by chest radiography (Figure 2).

The following night, the pigtail catheter was accidentally 


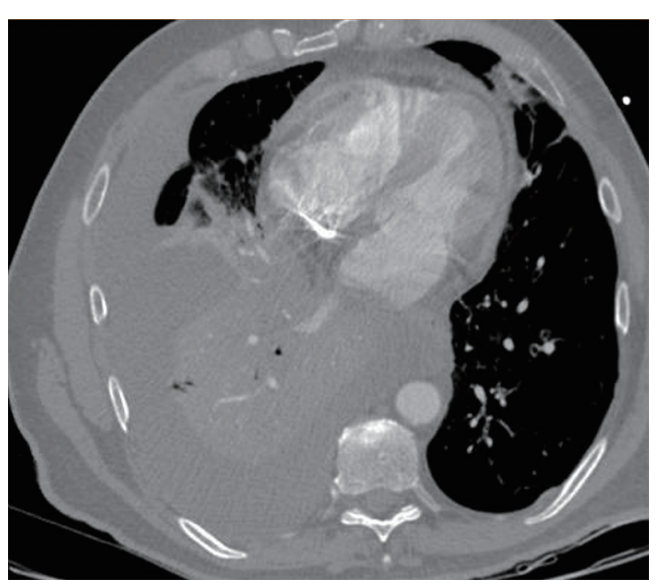

Figure 1 Axial image of chest CT: right pleural effusion with overlying compressive atelectasis and superimposed consolidation. Concentric pericardial effusion also seen.

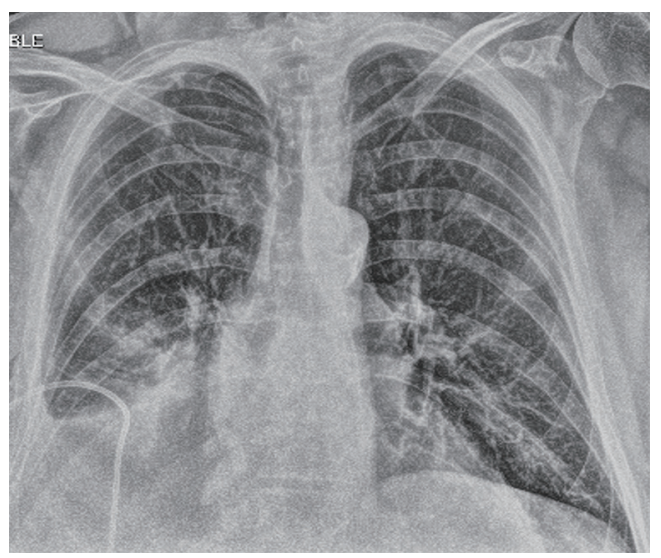

Figure 2 Posteroanterior chest radiograph: pigtail catheter seen in the right pleural space.

displaced while the patient was ambulating and was replaced with another one. Ultrasound-guidance was not employed. Chest radiograph performed the following day revealed near complete resolution of the pleural effusion but extensive subcutaneous emphysema was observed. A chest CT that was done to further investigate the findings on chest radiography showed the right pleural catheter with anterior entry point extending posteriorly through right middle and lower lobes, terminating in the posterior right pleural space. Also seen was interval development of pneumomediastinum and diffuse subcutaneous emphysema (Figure 3).

Subsequently, the patient underwent right video-assisted thoracoscopic surgery (VATS) for pleural biopsy and removal of the pigtail catheter under direct vision. Two

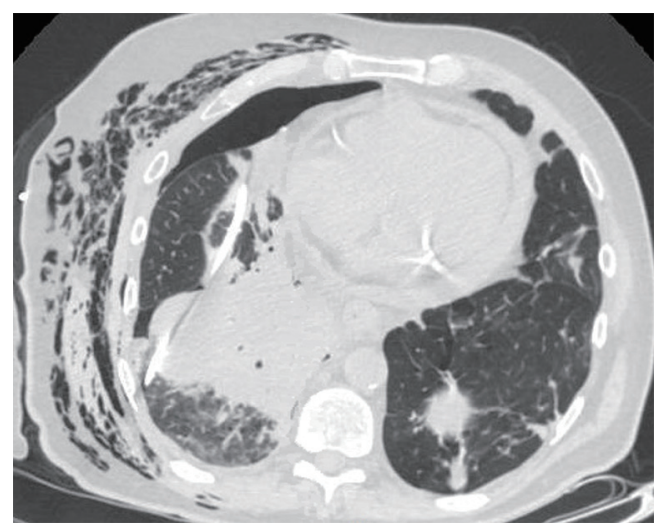

Figure 3 Axial image of chest CT: right-sided pigtail catheter with anterior entry point extending posteriorly through right middle and lower lobes, terminating in the posterior right pleural space. Also seen is pneumomediastinum and diffuse subcutaneous emphysema.

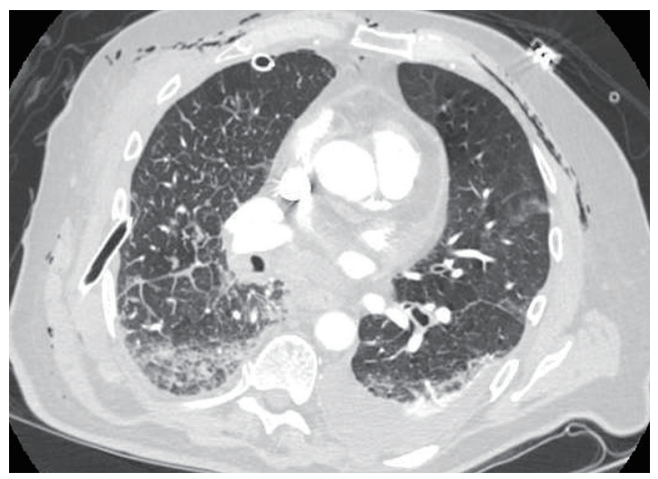

Figure 4 Post-VATS axial image of chest CT: complete resolution of the pneumothorax, subcutaneous emphysema and pneumomediastinum. VATS, video-assisted thoracoscopic surgery.

right-sided chest tubes were placed; one located overlying the right hemidiaphragm, and another one located along the medial superior aspect of the right hemithorax. Eight hours post-procedure, the patient developed pericardial tamponade requiring a pericardiocentesis. Pleural biopsy was consistent with adenocarcinoma of lung origin. The chest tubes were subsequently removed after complete resolution of the pneumothorax, subcutaneous emphysema and pneumomediastinum (Figure 4).

\section{Discussion}

Thoracostomy tubes are indicated for management of air or fluid in the pleural cavity. This can be achieved with a 
chest tube or pigtail catheter. Pigtail catheters differ from traditional chest tubes with respect to smaller bore and flexibility leading to lower rate of procedural complications and less ambulatory limitations (2).

The complications of tube thoracostomy may include pain, pneumothorax, hemothorax, infection, dislodgement, kinking, disconnection or blockage of the tube. Kinking and blockage occur more frequently with pigtail catheters given their smaller diameter (3). Certain rare complications have also been reported in the literature. Kim et al. reported a case of a 67-year-old man who developed impaired vision immediately after pigtail catheter insertion. Head CT findings were consistent with multiple cerebral air emboli (4). The air embolism by lung puncture is postulated to result from gas bubbles entering a pulmonary vein. There are several different possibilities for the mechanism of occurrence. First, air bubbles can flow in when a needle tip enters a pulmonary vein while its base is opened to the atmosphere. Once the atmospheric pressure exceeds the pulmonary venous pressure during inspiration, air enters the pulmonary venous system. Second, it may occur when a needle penetrates both an air-containing space and a nearby pulmonary vein, causing a bronchovenous fistula (4). Another case of cerebral air embolism that proved fatal was reported by Alkhankan et al. They describe a case of a 50-year-old man who became suddenly unresponsive and apneic followed by development of left-sided weakness during insertion of a pigtail catheter. CT angiogram was consistent with air emboli in the right main cerebral artery (5). Kim et al. reported a case of subclavian artery laceration during removal of the pigtail catheter that was inadvertently placed in the first intercostal space leading to massive hemorrhage and hypovolemic shock upon removal (6). With rapid administration of fluid, systolic blood pressure was scarcely maintained at $80 \mathrm{mmHg}$. A chest tube was placed in the left pleural space and three liters of fresh blood was drained initially. While waiting for emergent angiography, ventricular fibrillation occurred. The patient was successfully resuscitated in four minutes. Angiography showed extravasation of the dye from the left subclavian artery. To reduce blood loss before surgery, occlusion with a compliant balloon (13 $\mathrm{mm}$ in diameter) was made at the level of the left proximal subclavian artery following which the bleeding decreased. Primary repair of the left subclavian artery was attempted under general anesthesia in the operating room. A small hole of the left subclavian artery on the anterior side, at $5 \mathrm{~mm}$ apart from the thoracoacromial trunk, was found and primary suture repair of the injured subclavian artery was performed (6). Danekas et al. reported a potentially lethal complication of inadvertent placement of the pigtail catheter in the left ventricle in a 3-year-old patient resulting in pulsatile blood flow following advancement of the introducer needle. An emergent operation was done whereby a sternotomy was performed and the left pleura was opened. The catheter was visualized passing through a completely atelectatic left lower lobe and into the pericardium, just missing the phrenic nerve and circumflex coronary artery. A horizontal mattress pledgeted suture was placed surrounding the catheter which was removed and the suture tied (7).

Lung laceration is a relatively common complication of large bore chest tube placement (8). However, to our knowledge, lung penetration with a pigtail catheter has not been reported in the literature. In large bore chest tubes, the intraparenchymal insertion of the tube is usually associated with the use of a trocar for initial insertion (8). We believe that in our case, during the insertion of the replacement catheter when the guide wire was used to direct the catheter, it penetrated the lung parenchyma due to approximation of the lung to the pleura since most of the fluid had already drained. The penetration did not cause any immediate complications which led to a delay in diagnosis. The catheter tracked through the right middle and lower lobes eventually reaching the posterior pleural space leading to continuous drainage and failing to arise a suspicion of a procedural complication. The re-insertion of the catheter had been attempted without ultrasound guidance under the assumption that the original site of insertion is still ideal for the catheter without giving due consideration to the fact that a significant amount of fluid had drained since the original insertion. This highlights the importance of use of ultrasonography for the insertion of pig tail catheters. Image guided technique ideally ultrasound should be applied for pig tail insertion to minimize the risk for complications. British Thoracic Society recommends that ultrasound guidance is used if the effusion is very small or initial blind aspiration fails (9).

\section{Acknowledgements}

None.

\section{Footnote}

Conflicts of Interest: The authors have no conflicts of interest to declare. 
Informed Consent: Witten informed consent was obtained from the patient for the writing of this case report.

\section{References}

1. Gammie JS, Banks MC, Fuhrman CR, et al. The pigtail catheter for pleural drainage: a less invasive alternative to tube thoracostomy. JSLS 1999;3:57-61.

2. Lin CH, Lin WC, Chang JS. Comparison of pigtail catheter with chest tube for drainage of parapneumonic effusion in children. Pediatr Neonatol 2011;52:337-41.

3. Horsley A, Jones L, White J, et al. Efficacy and complications of small-bore, wire-guided chest drains. Chest 2006;130:1857-63.

4. Kim SI, Kwak HJ, Moon JY, et al. Cerebral air embolism following pigtail catheter insertion for pleural fluid drainage. Tuberc Respir Dis (Seoul) 2013;74:286-90.

Cite this article as: Saqib A, Ibrahim U, Maroun R. An unusual complication of pigtail catheter insertion. J Thorac Dis 2018;10(10):5964-5967. doi: 10.21037/jtd.2018.05.65
5. Alkhankan E, Nusair A, Mazagri R, et al. Systemic Air Embolism Associated with Pleural Pigtail Chest Tube Insertion. Case Rep Pulmonol 2016;2016:4053748.

6. Kim JH, Cho YH, Suh GY, et al. Subclavian Artery Laceration Caused by Pigtail Catheter Removal in a Patient with Pneumothorax. Korean J Crit Care Med 2015;30:119-22.

7. Danekas MG, Dietrich PP. Unusual Complication of Left Pigtail Chest Tube Insertion. Society of Pediatric Anesthesia, meeting abstract, 2010.

8. Remérand F, Luce V, Badachi $Y$, et al. Incidence of chest tube malposition in the critically ill: a prospective computed tomography study. Anesthesiology 2007;106:1112-9.

9. Laws D, Neville E, Duffy J. BTS guidelines for the insertion of a chest drain. Thorax 2003;58:ii53-9. 